Published in: The Nature of Time: Geometry, Physics and Perception. R. Buccheri, M. Saniga and W. M. Stuckey, eds. NATO Science Series II. Mathematics, Physics and Chemistry - Vol. 95, Kluwer Academic Publishers, Published in Netherlands, 383-392, 2003.

\title{
THE DYNAMICS OF TIME AND TIMELESSNESS: PHILOSOPHY, PHYSICS AND PROSPECTS FOR OUR LIFE
}

\author{
ATTILA GRANDPIERRE \\ Konkoly Observatory \\ H-1525 Budapest, P. O. Box 67, Hungary
}

Keywords: endo-physics - ontological levels - integrated ontology - ultimate time

\section{The Ultimate Nature of the Universe}

\section{1. ENDO-PHYSICAL, EXO-PHYSICAL AND ULTIMATE TIME}

The concept of endo-physics was introduced [1-2] in the context of self-reference in quantum physics and artificial intelligence (AI) research. Now, regarding quantum physics, it implicitly relies on something existing outer to itself, namely, the existence of an observer related to consciousness. In order to evaluate the relation of consciousness to (quantum) physics, it is necessary to recognise the fact that physical behaviour follows the principle of least action (action principle) which requires the behaviour to be directed towards the physical equilibrium. Now since biology follows a principle which acts against the physical principle, attempting to preserve the distance from physical equilibrium, and consciousness allegedly develops only in biological organism (at least in the observer concept of quantum physics), the observer lives on an ontologically different level from the physical realm [3-5]. Therefore, the epistemological structure of the observer-observed relation contains a two-levelled ontological structure. Regarding AI, it relies to "creators" of hardware and software, therefore its epistemology is that of the deistic materialism [6], i.e. another ontologically two-levelled structure. Now self-modification, the concept closely related to endophysics [7], is characteristic on a biological and/or psychological level of ontology [4-5]. Therefore, it is an important recognition that self-governing systems follow developmental paths different from physical entities. Therefore endo-physics, putting the observer into a physical context compresses the two-levelled ontological structure into a monistic ontology and therefore creates epistemic paradoxes with Klein-bottle structures. Nevertheless, when we are aware that there is an at least two-levelled ontology behind the concept of endophysics, it is easy to remove certain types of paradox epistemic structures like Klein-bottles since self-reference becomes simply reference of one existential level to another.

It was shown already by Aristotle [8] that the psyche appears to be responsible for the "measuring" and "the counting" of (physical) motions and thus, for the coming into being and the genesis of chronos at the human level as a psychological phenomenon with ontological pretensions. This notion is close to the one expressed in Boltzmann's Htheorem that entropy is generated only in measurements (see also Eakins, this workshop). Recognising the two(or three)-levelled ontology (physical-biological-noetic) behind the 
endo-physical concept, it is easy to escape from the epistemic paradoxes of endo-physics [9]. Self-reference in a physicalist epistemology should form a Klein-bottle structure and Klein-bottles cannot be factually built. In a two(or three)-levelled ontology observercontaining modelling requires only the reference of the higher (cognising) ontological level to the lower (physical) level and so the epistemic structure is a simple mapping relation. Therefore the claim of [9] that a new ontology may be necessary is true already at the introduction of the concept of endo-physics.

The physical view - in contrast to the endo-physical view - requires the ignorance of human (and, in general, non-physical) views. At the same time it is a materialistic, inanimate view, and therefore it is bound to some finite relations between inanimate systems. Therefore, physical view is outer in comparison to our internal view, but it is internal within the materialistic systems of relations. Apparently, while it expresses an unnatural, non-realisable view, it ignores the connection to the source of genuine activity of the material world.

The endo-physical view relies on an internal, psychological, mental or biological view. The concept of endophysics is an ancient one. Spinoza [10] recognised that an internal being can grasp the nature of the parts if it moves not from the part to the whole but from the whole to the part. Grandpierre [3] suggested that an internal being may recognise the nature of the whole by studying the most general relations, which are the ultimate, ontological principles. To recognise the ultimate (exo-cosmic) view, we should comprehend the Universe as a whole. There is more to reality than that meets the eye. The ultimate view regards the Universe as a unified organisation of three ultimate realities: they are the realms of the material (physical), the emotional (biological) and the thought-like (psychological) existents. Therefore, the ultimate view is closely related to the recently analysed three ultimate principles [4-5]: that of physics, biology and psychology. It points out that matter is a manifestation of the material principle, which is the action-principle of physics.

\section{2. THE TIMELESSNESS OF THE UNIVERSE}

The Universe when defined as an all-inclusive notion [3] is time-inclusive, too. In this view time is an internal part of the Universe, and the Universe-as-a-whole is living behind the temporal realms, therefore, in timelessness. Apparently, the realm of the $3+1$ dimensions is related to thermodynamics. Unfortunately, the law of entropy development is not proven for all systems, especially not when gravitational, electromagnetic, weak, strong and other (possibly biological) interactions play a significant role. It is already indicated that some interactions may be related to compactification of higher dimensions. Since biology is able to govern behaviour, biology is able to govern physics. We can expect that the biological principle, representing a subtle, biological interaction, may belong to some higher-thanphysical dimension and/or to the ultimate, logical dimension, which is behind the finite spaces.

\section{3. HIGHER AND ULTIMATE DIMENSIONS AND BEHAVIOUR}

It is well known (e.g. [11]) that the spatio-temporal characteristics of the so-called "extraordinary/altered" states of consciousness may profoundly differ from our everyday experience. To grasp the basic structure of these "anomalous/peculiar" space-times, it seems to be necessary to go beyond the mathematical framework offered by physics. The 
proper understanding of such "uncanny" space-times was demonstrated to require highly abstract geometrical concepts like projective planes over finite (Galois) fields [12-13], and/or Cremona transformations [14-15]. Moreover, not only the nature of a mental state seems to be related to a special mathematical space, but it is frequently reported that travel between the different spaces and times is possible by will. The question arises as which kind of factor regulates the $3+1$ and higher dimensions. In the context that physics is based on the ultimate principle of least action, which is related somehow on a virtual transparency of the $3+1$ dimensional space as a whole, we raise up the possibility that the higher dimensions are more related to the biological and spiritual principles besides of the physical principle.

\section{On the Nature of the Ultimate Dimension}

\section{1. SOUL AS A FIRST PRINCIPLE}

Analysing the concept of "soul" it is found [5] that in some ancient high culture the soul is conceptualised as the ultimate driving factor of life. The Dictionary of Hungarian Language [16] determines the concept of the soul as the following: " $1 .<$ By a primitive $>$ concept the soul is the hypothesised, more-or-less material ultimate carrier of life phenomena, which departs the body at the moment of death". At the same time, a closer scrutiny reveals that this allegedly "primitive" conceptualisation is related to the deepest scientific concept of mankind, which is the concept of "first principles". Eisler [17] stated that soul appeared as a (first) principle at the special kind of animism of ancient Greek philosophers.

Scientific research attempts to reveal facts and deeper relations. Science begins when we search the laws behind the phenomena. Now laws may be regarded as deeper level relations behind the immediate, brute facts. Although laws help us to explain and predict phenomena, they may be regarded as being only the first steps on the way to find the most clear and most transparent truth possible, which is the ultimate aim of science. Therefore, the real basis of science is related to the laws behind the laws, and to find the ultimate law which is able to explain all the laws intermediate between empirical facts and mental understanding. Now the concept that developed the notion of ultimate and universal laws, the first principles, may be regarded as the highest point of scientific conceptualisation. Therefore, soul as a universal first principle, as an ontological principle is a scientifically remarkable concept from which one can expect fundamental insights into the Nature.

\section{2. PRINCIPLES, PRE-REALITY AND POTENTIALS}

The action principle of physics is a universal and ultimate principle, an arche. Therefore, the materialised universe may be regarded as governed by a primary factor, the arche. In this way time seems to be a secondary attribute of the primordial, timeless reality. The ultimate principles act in the logical dimension, from "above" time and space, therefore they may transcend space and time. The archi may act as primary potentials, or pre-existent realities, pre-realities. Being pre-material, their action is not a solidified material action. Being pre-time existents, they may exert integral organisational influence and determine the path through which the material process should be realised. These requirements may be related to the virtual paths in the Feynman path-integral at the calculation of the actual path realised under the action principle. The primary potentials may be related to such integral 
phenomena as the primal interaction [18], the phenomenon of pre-lightning [19], at the pattern formation of Benard-cells [5], spontaneous targeting of enzymes, at the reaction kinetics of the hydrogen-oxigen mixture burning [20] etc.

The biological principle is indicated to act behind (or before?) any space and time, in the ultimate reality. In this context the psychic reports on some mathematical details of higher spaces may reveal us the fact that the higher spaces are under a (partial or dominant) psychic control, and so the phenomena are not governed by physics alone, but also and (perhaps prominently) by biology. My proposal is that in these contexts the physics of higher spaces may be complemented by the ultimate principle of biology.

\section{3. THE LIFE PRINCIPLE}

In the biological context the behaviour is governed by the life-principle, as determined by Bauer [21]. "Living systems are characterised by the fundamental and general law, telling that the living system exerts work against the realisation of the equilibrium...changing the internal conditions, therefore its own structure. If the living system is effected to an outer influence and it exerts a work, this work has to modify the effect of the outer influence in order to increase the ability of the system to work." The ultimate principle of biology is formulated by Ervin Bauer in the following way: "The living and only the living systems are never in equilibrium, and they continuously work supplied by their free energy content against the realisation of the equilibrium which should set up in the same outer conditions by the laws of physics and chemistry".

\section{4. TRANSPARENCY}

Regarding the fact that the biological principle is able to govern the behaviour, physical causation is recognised to be open towards the ultimate dimension. This fact makes the regulation of an organisation possible in an extremely transparent manner. The extreme richness and complexity of biological organisation requires the presence of an integral, simultaneous, but also a regulative mechanism potentially infinitely extended in time. This type of transparency is not available in the $3+1$ dimension since material objects represent a kind of shielding. Interestingly, the higher dimensions offer a kind of transparency unprecendented within the 3+1 material dimensions. Moreover, the higher and the ultimate dimensions carry additional degrees of freedom and related additional information which may be important at the organisational activities.

\section{Our Life in the Ultimate Cosmic Evolution}

\section{1. HIGHER CONTEXTS OF OUR LIFE}

The ultimate view of the Universe is in a close relation with the timeless character of our thoughts and emotions. The aim to give our life a meaning exerts important influence on our life as a whole, which does not pass with the end of our earthly life. We should not regard as true the widespread materialist view that our life is born from inanimate matter, and we will return to inanimate matter (from dust to dust), into the complete annihilation. It is not true that we should conceive our life in a sport language that before our birth the 
game had a state $0: 0$, during our life the state is $1: 0$, and after our life it is $1: 1$, and this is completely the same state as before our birth. This view is not true because the fact of our life as a whole will not become invalid by our death. The fact of our death cannot change the validity of the fact that we had a life. Therefore the fact of our life is a member of a larger context and forms a part of it. One part of the higher contexts concerning our life is the survival of the aim of our life, a maintenance and further development of the values which were important to our life. This part of our life is related to our culture. The other part of the higher contexts of our life is directly related to the Universe as a whole. This is because our thoughts and emotions are not extended in space and time (they become extended in these dimensions only through their travel to our outer world). Therefore they have the same nature as the Universe as a whole, and so our thoughts and emotions are added to the Universe as a whole in the logical field of the Universe. This logical field is a reason-full natural system and has a timeless nature. Our thoughts and emotions may gain directly cosmic significance in the extent as they connect to the logical and reason-full, real order of the Universe. The thoughts and emotions born by our life may enter directly to the timeless Universe and there their existence may gain a meaning and significance in a logical form, in a timeless essence. Logic is more than individual consciousness - it is a potential of infinite, relevant and true consciousness and creativity. Logic is the cosmic network of the primal, pre-material, biological and psychological existents. In so far as our thoughts and emotions carry a meaning that ignores the meaning and destination of the Universe, this timelessness will have a different context and significance than the thoughts and emotions helping the realisation of the destination of the Universe.

\section{2. ETERNAL INVARIABILITY OF THE UNIVERSE: THE THREE ARCHI}

Since we were able to obtain an insight into the life centre of the Universe when we recognised the three archi, we have a possibility to consider a not yet suspected cosmic connection of Eternity and terrestrial life. If the Universe has a principal nature, and the principles have a spiritual, mental, universal, cosmic nature above space and time, the principles should live also above space and time, and so they extend to time as a whole. Now we should face with the question: how could we conceive the destination of the Universe in a timeless context? If any evolution should occur in time, and the nature of the Universe is timeless, does not this fact involve that the Universe is destined to be forever changeless? And will not an eternal changelessness connected necessarily with a meaninglessness - since eternal invariability is interpreted in a material view as a state in which nothing to do?

\section{3. OUR THOUGHTS AND EMOTIONS AND THEIR TIMELESSNESS}

In contrary to this possible objection, if our thoughts and emotions born by our life add continuously to the logical network of the Universe, than the Universe is necessarily destined to a kind of evolution. This evolution is an interesting, extraordinary and unsuspected one. This evolution starts from realms of time and arrives to the realm of timelessness and completes its ultimate meaning in timelessness. One can clearly observe that with the help of life timelessness obtains a possibility to evolve. But what is the nature of the time in which timelessness evolve? This ultimate time should be different from our everyday, material-contexted time. More exactly, we live our everyday life also in timelessness through our thoughts and emotions, but this fact did not become realised, and 
it is a general view that we live our life exclusively in material-contexted time. Evangeliou [8] pointed out that Aristotle, following Plato, connected time to the Cosmic Soul and conceived of it as an "image of eternity", in relation to the Divine Nous and the "Kosmos Noetos". At the same time, peak-experiences, near-death experiences show that we can live during our life also with the abilities offered by higher dimensions and the ultimate reality. The ultimate view of time shows that we can live our life in its full scope when we live with the power of our ultimate reason.

\section{4. THE TIME IN WHICH TIMELESSNESS EVOLVES: LOGICAL CONTEXT}

How could we conceive the time in which timelessness evolve? If we imagine the three arche forming an organic, alive whole as three red threads running from infinity to infinity, then these three threads may develop in the reason-full completion of the Universe. When we have to speak on the ultimate scene we should apply some parable which is closer to the material view. With the help of a parable, in which three red threads represent the three archi, the evolution in the timeless sphere may be imagined as a fourth thread developing above and around these already existing three threads, unifying them as a metaphysical current circuit. And since this new thread is born and developing in the principal level, its evolution does not met with any constraint. In the principal level life is a full, absolute intensity, an unconstrained reality. Therefore, all newborn existent having principal significance has to build in into the principal existence. Finite, limited, bordered existence and infinite existence forms a dynamic dyad. Unlimited existence knows everything but still incomplete since it misses finiteness. Unlimited principal existence can realise everything, every wish, desire, aim immediately, therefore there can be no space and no time at this level of existence. But because unlimited infinite existence still misses finiteness, its completion needs the realisation of finiteness. Finiteness knows something more about existence, because it is coupled with the concept of stake. In finiteness one can occur something which is unavailable in infinity: it is selection, purification, a game which goes for life and death, a game in which everything is concentrated in a stake.

\section{5. THE DYNAMICS OF FINITE AND INFINITY IN OUR LIFE}

The ultimate concentration of infinity in finiteness is called life, the ultimate stake. Therefore, when our life is at risk, it is the concentrated infinity which is at risk, which is under the attack in the finite existence. The dynamics of finiteness and infinity is paralleled in the dynamics of timely and timeless existence. Material reality is not a separate, isolated subset of existence, which is closed in itself. Material reality is related with the realities of life and reason. Material reality forms a complete reality with the realities of life and reason. This complete reality has a principal, spiritual nature. In this way it is necessary that our thoughts and emotions should form a communicating unity with each other and the material reality. In this way, the destination of the Universe requires a development, an evolution in a more full sense, the time of which is the logical time, the time of reason, the order of the completion of the reason-full, genuine, cosmic meaning.

\section{6. THE DYNAMICS OF SPIRITUAL PRINCIPLES AND INERT MATTER EMERGENCE}


Von Neumann estimated that the critical number of elements necessary in a system to be self-reproducible is around $10^{6}$ [22]. "There is thus this completely decisive property of complexity, that there exists a critical size below which the process of synthesis is degenerative, but above which the phenomenon of synthesis, if properly arranged, can become explosive, in other words, where synthesis of automata can proceed in such a manner that each automaton will produce other automata which are more complex and of higher potentialities than itself." This statement is based on the accumulative and explosive increase of $(\mathrm{k}$ under $\mathrm{N})(\mathrm{k}=1,2, \ldots, \mathrm{N})$ with growing $\mathrm{N}$. Therefore, the automaton cannot produce a higher complexity automaton, without being absolutely creative and able to determine the arrangement which leads to a working automata with reasonable functions. This condition is a requirement of a foreseeing and evaluating the arrangements in a creative manner. Nevertheless, Neumann's argument may be applied to living systems which have an access to a creative ultimate principle like the biological principle. "There is a minimum number of parts below which complication is degenerative, in the sense that if one automaton makes another the second is less complex then the first, but above which it is possible for an automaton to construct other automata of equal or higher complexity. Where this number lies depends upon how you define the parts. I think that with reasonable definitions of parts, like those I will partially indicate later, which give one or two dozen parts with simple properties, this minimum number is large, in the millions" [22].

Now let us assume that there are only one type of constituents in the central nervous system, neurons. In order to arrange them into creative units able to produce combinations in a larger set of possibilities than they are made of, a critical number of nerves should be around million. Assuming that in such a nervous system an integral macro-unit will form which acts as an agent, we may think about the necessary condition of self-consciousness. Self-consciousness is approached here in the context being realised by a macro-micro coupling acting above the macrolevel of consciousness that is reached by a lower level macro-micro coupling [18]. Representing the integral unit with a central unit, with a growing number of brain elements the number of central units may grow as well. Applying again the Neumann-criterion, we may obtain that the necessary number of central units may be around a million and this may be represented with an integral unit of central units. In this case, when each central unit represents a million nerves, the integral unit of central units may represent a thousand billion neurons, which is interestingly quite close to the number of nerves, around 100 billion, in human brain, which we know as being able to selfconsciousness.

\section{Ontological Principles as Elements of the Bridge through the Pythagorean Gap}

In the approach outlined in this work the necessity of an ultimate, integral cosmology is indicated in which the principles of biology (and psychology) should be also implemented besides the physical principle. The evolution of the Universe occurs in the logical time of Eternity. We can contact with Eternity if we are able to connect our emotions and thoughts into the reason-full, logical order of the Universe. There is no time without reality. Reality is founded ultimately on the ontological levels. The interaction of the ontological levels creates the logical time of Eternity. It is an interesting question how the ontological principles are build up from the logical principles. Pythagoras expressed the view that physical reality is generated from the realms of mathematics. Today, as we understand it, there is a gap between the mathematical (logical) and physical (material) levels of 
existence. This Pythagorean Gap connects the realms of mathematics and physics, infinity and finiteness, being and knowledge. We found at the origin of material time the realm of logic. Regarding the ontological principles as built up in the realm of logic, it is promising to explore their mathematical construction and formulation. The ontological principles may become important elements in bridging up the Pythagorean Gap.

\section{Acknowledgement}

The author wishes to express his gratitude to Prof. Bela Lukacs for a careful proofreading of the paper.

\section{References}

1. Finkelstein, D., Finkelstein, S. R. (1983) Computational Complementarity, Int. J. Theor. Phys. 22: 753-779.

2. Rössler, O. E. (1987) Endophysics, in: Real Brains, Artificial Minds (eds. J. L. Casti, A. Karlquist), NorthHolland, New York, 25-46.

3. Grandpierre, (2000),The nature of the Universe and the ultimate organisational principle, Ultimate Reality and Meaning, 23: 12-35.

4. Grandpierre, A. (2002a) The fundamental principles of existence and the origin of physical laws, Ultimate Reality and Meaning, 25: 127-147.

5. Grandpierre, A. (2002b) The Book of the Living Universe (in Hungarian).

6. Smart, J. J. C. (1989) Materialism, in: The New Encyclopedia Britannica, Enc. Br. Inc., 25: 630-634.

7. Kampis, G. (1990) Self-Modifying Systems in Biology and Cognitive Science, Pergamon Press, Oxford.

8. Evangeliou, C.( 2002, this volume) The Aristitelian Realtion of Time to Motion and to Soul

9. Kampis, G. (1994) Biological Evolution as a Process Viewed Internally, in: End/Exo Problems in Dynamical Systems, H. Atmanspacher, G. J. Dalenoort (eds.) Springer, Berlin.

10. McIntyre, A. (1967) Spinoza, Benedict (Baruch), in: The Encyclopedia of Philosophy, P. Edwards, editor-inchief, The Macmillan Co. and The Free Press, New York, 7: 530-541.

11. Jaspers, K. (1923) Allgemeine Psychopathologie, Spriner, Berlin.

12. Saniga, M. (1998) Unveiling the nature of time: Altered states of consciousness and pencil generated spacetimes. The International Journal of Transdisciplinary Studies. 2: 8-17.

13. Saniga, M. (1999) Algebraic geometry: a tool for resolving the enigma of time? In: Studies on the structure of time, eds. Buccheri, Di Gesu, and Saniga, 137-166

14. Saniga, M. (2001) Cremona transformations and the conundrum of dimensionality and signature of macrospacetime, Chaos, Solitons \& Fractals Vol. 12, 2127-2142.

15. Saniga M. (this volume) Geometry of time and dimensionality of space.

16. The Dictionary of Hungarian Language (1979) lélek, Akadémiai Kiadó, Budapest, IV: 702 (in Hungarian).

17. Eisler, R. (1927) Animismus, in: Vörterbuch der Philosophischen Begriffe, E. S. Mittler \& Sohn, Berlin, 1: 52

18. Grandpierre, A. (1999) The Nature of Man-Universe Connections, The Noetic Journal, 2: 52-66.

19. Feynman, R. P., Leighton, R. B., Sands, M. (1963) The Feynman Lectures in Physics, Addison-Wesley Publ. Co., Reading, MA.

20. Hemley, R. J. (1995) Turning off the Water, Nature 378: 14

21. Bauer, E. (1967) Theoretical Biology, in Hungarian (1935, in Russian).

22. Neumann, John von (1966) Theory of Self-Reproducing Automata, edited and completed by A. W. Burks, University of Illinois Press, Urbana and London, 80. 